\title{
A Case of Leptomeningeal Dissemination of Pilocytic Astrocytoma in a Child
}

\author{
Yu-Feng Su, Chih-Hui Chang, Shyh-Shin Chiou, Chee-Yin Chai, Shiuh-Lin Hwang, \\ Joon-Khim Loh
}

\begin{abstract}
We present the case of a 2-year-old boy with progressive left-sided weakness and a cranial magnetic resonance imaging (MRI) scan showing a lesion with a cystic component in the right thalamus and basal ganglia. The lesion was subtotally resected and diagnosed as a pilocytic astrocytoma by histopathology. Tumor seeding along the surgical tract was seen on MRI 16 days and 10 weeks after surgery. The patient received vincristine and carboplatin, and MRI performed 4 months after chemotherapy revealed no additional or residual lesions. This case illustrated that a World Health Organization grade I astrocytoma could disseminate along the surgical tract.
\end{abstract}

RÉSUMÉ: Nous voulons présenter le cas d'un garçon de deux ans chez qui on a diagnostiqué un déficit partiel de la force musculaire du côté gauche. Un examen de tomodensitométrie (ou IRM) a aussi montré chez cet enfant la présence d'une lésion kystique du côté droit du thalamus et dans les ganglions de la base. Cette lésion a été ensuite partiellement reséquée; de plus, une histologie pathologique a révélé qu'il s'agissait d'un astrocytome pilocytique. La dissémination de la tumeur cancéreuse le long de l'incision chirurgicale (surgical tract) a été observée au moyen d'une IRM 16 jours après l'opération chirurgicale puis 10 semaines après cette dernière. On a alors administré au jeune patient un traitement de chimiothérapie à la vincristine et au carboplatine. Une IRM effectuée 4 mois après ce traitement n'a révélé aucune lésion résiduelle ou additionnelle. Ce cas illustre donc dans quelle mesure un astrocytome de grade I (selon l'OMS) peut se disséminer le long de l'incision chirurgicale.

Keywords: brain tumor, leptomeningeal dissemination, pilocytic astrocytoma, tumor seeding

doi:10.1017/cjn.2017.22

Can J Neurol Sci. 2017; 44: 607-610

Pilocytic astrocytoma (World Health Organization grade I) has a benign biologic behavior, and one of the highest survival rates of any type of astrocytoma. However, it occasionally exhibits an atypical behavior characterized by local recurrence, or, in rare cases, malignant transformation and metastasis. ${ }^{1-3}$ Leptomeningeal dissemination of a pilocytic astrocytoma in children, especially young children, is very rare. ${ }^{1-3} \mathrm{We}$ describe a case of a pilocytic astrocytoma with leptomeningeal dissemination in a young boy.

A 2-year, 9-month-old boy was admitted in 2007 with a 6-month history of progressive left-sided weakness. Examination revealed left-side hemiparesis with partial pseudobulbar palsy, increase tendon reflexes in his left limbs, and a positive Babinski sign on the left. Cranial magnetic resonance imaging (MRI) scans disclosed a solid, well-circumscribed, contrast-enhancing mass in the right thalamus and basal ganglia, with a cystic component (Figure 1). Gadolinium-enhanced $\mathrm{T}_{1}$-weighted axial (Figure 1A) and coronal (Figure 1B) images revealed a nonenhancing cystic mass with an intensely enhancing mural nodule $(7.9 \times 5.4 \times 6.4 \mathrm{~cm})$ over the right basal ganglion and thalamus. A subtotal resection was chosen over gross total resection because the location of the tumor in the thalamus is an eloquent area. A repeat MRI was performed immediately after surgery to serve as the basis for future comparison.

The patient's postoperative course was unremarkable, and he could walk without assistance. Gadolinium-enhanced coronal $\mathrm{T}_{1}$-weighted MRI 16 days after surgery (Figure 1C) showed diffuse enhancement not seen in the MRI scans obtained immediately postoperatively, indicating possible tumor seeding along the surgical tract with extension into the subdural space. Residual tumor in the thalamic area was also noted. Reoperation was not considered because the areas of seeding were inoperable. No seeding of the spinal leptomeninges was noted in the spine MRI scan. Repeat MRI performed 10 weeks postoperatively and immediately before the start of chemotherapy showed similar results (Figure 1D, E).

Histopathological examination of the surgical specimen showed a moderately cellular glial tumor with spindle-shaped cells arranged in bundles. A biphasic pattern of loose glial tissue and compact piloid tissue was noted (Figure 2A). The piloid tissue component was composed of fusiform cells with wavy fibrillary processes and Rosenthal fibers, and the tumor was positive for glial fibrillary acid protein and vimentin, indicating astrocytic origin (Figure $2 \mathrm{~B}, \mathrm{C}$ ). The Ki-67 index was $2 \%$ to $3 \%$ (Figure 2D). Based on the histopathological examination, the final diagnosis was pilocytic astrocytoma. ${ }^{4}$

From the Division of Neurosurgery, Department of Surgery, Kaohsiung Medical University Hospital, Kaohsiung, Taiwan (YFS, CHC, SLH, JKL) Faculty of Medicine, Graduate Institute of Medicine, College of Medicine, Kaohsiung Medical University, Kaohsiung, Taiwan (YFS, JKL) Department of Pediatrics, Kaohsiung Medical University Hospital, Kaohsiung, Taiwan (SSC) Department of Pathology, Kaohsiung Medical University Hospital, Kaohsiung, Taiwan (CYC); and Kaohsiung Municipal Hsiao-Kang Hospital, Kaohsiung, Taiwan (JKL).

Received July 4, 2014. Final Revisions Submitted June 22, 2016. Date of ACCEPTANCE: August 9, 2016.

Correspondence to: Joon-Khim Loh, Division of Neurosurgery, Department of Surgery, Kaohsiung Medical University Hospital, 100 Tzyou 1st Road, Kaohsiung 807, Taiwan. E-mail: jokhlo@kmu.edu.tw 

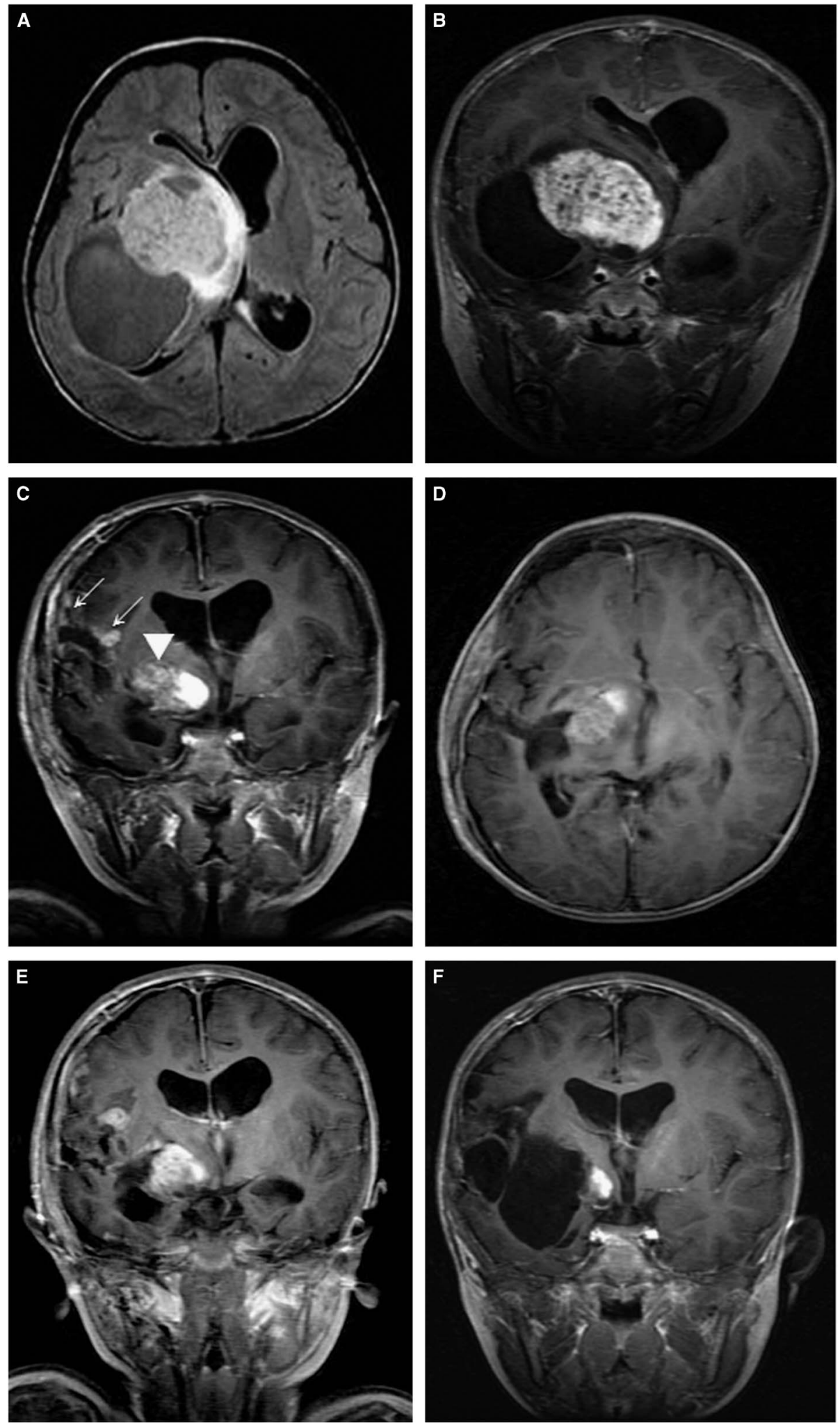

Figure 1: (A) Gadolinium-enhanced axial $T_{1}$-weighted (A) axial and (B) coronal MRI scans of a nonenhancing cystic mass with enhancing mural nodule over the right basal ganglion and thalamus. (C) MRI taken 16 days after surgery showed diffuse enhancement (arrows) and tumor tissue along the surgical tract, with extension into the subdural space, and residual tumor in the thalamic area (arrow head). (D) Axial and $(E)$ coronal $T_{1}$-weighted MRI of the brain immediately before chemotherapy. $(F)$ MRI performed 4 months after completion of chemotherapy revealed calcification, and no residual disease or additional lesions. 

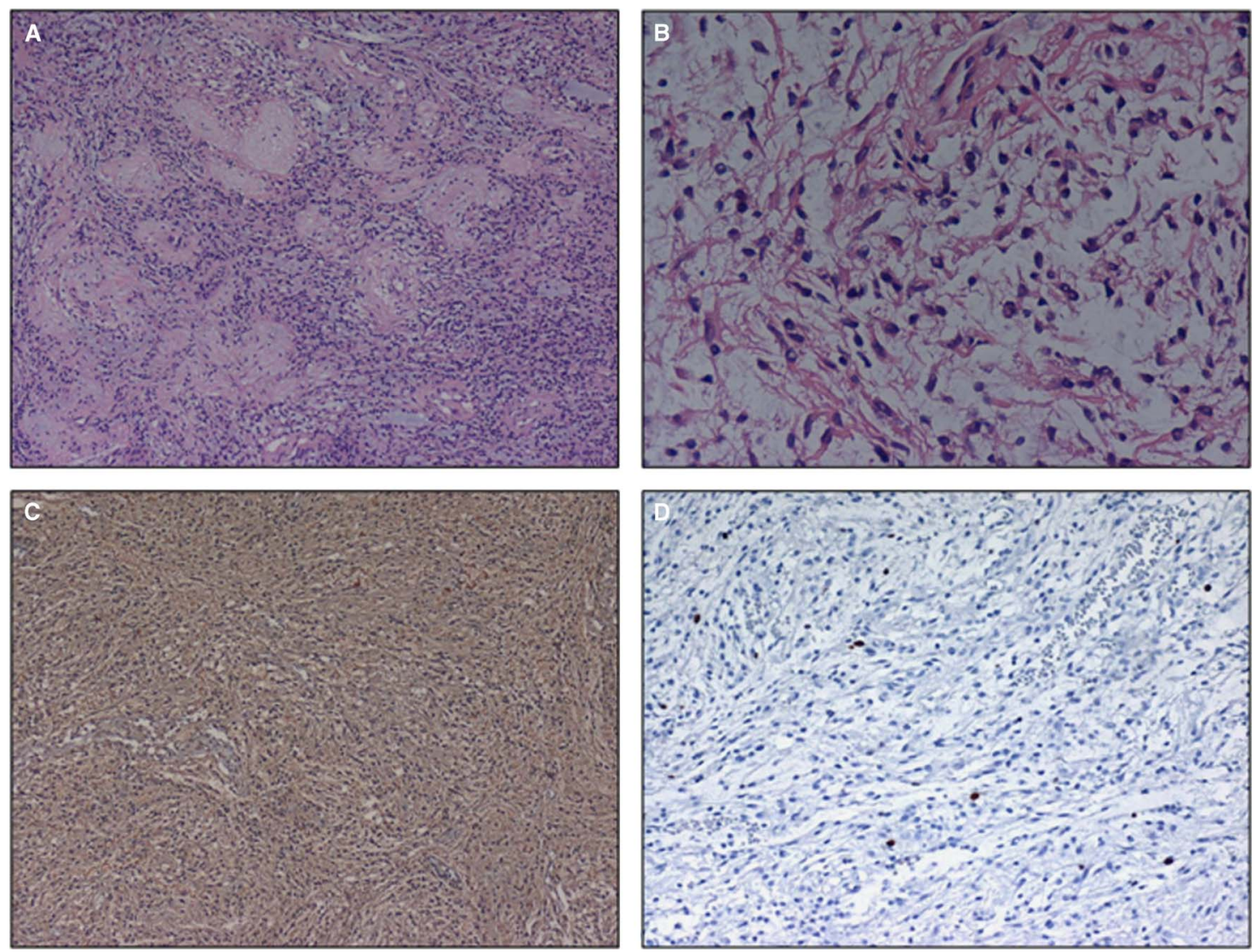

Figure 2: (A) Histological examination showed a biphasic pattern of loose glial tissue and compact piloid tissue. (B) The piloid tissue component was composed of fusiform cells with wavy fibrillary processes and Rosenthal fibers. (C) Tumor cells were diffusely positive for glial fibrillary acidic protein (immunostain, $100 \times$ ). (D) Ki-67 index was $2 \%$ to $3 \%$.

The patient received a six-course regimen of vincristine $\left(1.5 \mathrm{mg} / \mathrm{m}^{2}\right)$ and carboplatin $\left(175 \mathrm{mg} / \mathrm{m}^{2}\right)$. MRI performed 4 months after completion of chemotherapy revealed only calcification in the medial margin, but no residual or additional lesions (Figure 1F). No recurrence was noted in the subsequent 6 years of follow-up.

Leptomeningeal dissemination of pilocytic astrocytoma is uncommon, and as there have been only a few cases reported, its incidence is undetermined. In a review of the literature, we identified 29 cases of tumor recurrence/metastasis attributed to tumor seeding along the surgical, biopsy, or the aspiration tract, and five were malignant anaplastic astrocytomas or glioblastoma multiforme, unlike the relatively benign astrocytoma in our case. In more than half of cases (52\%), recurrence or metastasis was not detected until more than 1 year after the invasive intervention, with the longest interval being 21 years. It is estimated that $5 \%$ of all pediatric low-grade gliomas have leptomeningeal dissemination at diagnosis, and $7 \%$ to $10 \%$ have dissemination at the time of progression. ${ }^{5}$ Studies have reported an incidence of dissemination ranging from $4 \%$ to $12 \%$. $^{1,3}$

Specific extracellular substrates such as proteins, glycosaminoglycans, parenchymal cells, adhesion molecule production, protease secretion, growth factor pathway activation, immunogenicity of the tumor cells, and mechanical factors can all aid the leptomeningeal spread of pilocytic astrocytomas. ${ }^{2,3}$ Surgical manipulation can also promote dissemination. ${ }^{1}$ Patients with dissemination from a pilocytic astrocytoma may remain asymptomatic and survive for a long period. ${ }^{1,2}$
Complete resection can be curative in pediatric patients with low-grade gliomas, with a survival rate of more than $90 \%$ at 10 years. ${ }^{6}$ If only partial excision can be performed, chemotherapy may be recommended, especially if the patient is younger than 3 years of age. ${ }^{7}$ Radiation therapy is not typically used in younger patients because it can produce long-term neurocognitive and endocrine toxicity. Genkow et $\mathrm{al}^{6}$ reported that dissemination, age $<1$ year, and nonpilocytic histology were unfavorable factors for progression following radiotherapy, and diencephalic syndrome, dissemination, and age $\geq 11$ years were unfavorable factors following chemotherapy. Ater et $\mathrm{al}^{8}$ found that factors independently predictive of worse survival were younger age, tumor size $>3 \mathrm{~cm}^{2}$, and tumor location in the thalamus. In summary, the case reported provides neuroimaging and pathological findings of a pilocytic astrocytoma in a 2-yearold child with dissemination characteristics more commonly seen in World Health Organization grade III or IV astrocytomas. Neurosurgeons operating on patients with these tumor types should be aware of the possibility of tumor seeding along the operative tract. Even for a low-grade astrocytoma, as in our case, imaging at regular intervals after surgery to screen for tumor seeding along the surgical tract can be critical to the final outcome.

\section{DISCLOSURES}

The authors do not have anything to disclose. 


\section{REFERENCES}

1. Mamelak AN, Prados MD, Obana WG, Cogen PH, Edwards MS. Treatment options and prognosis for multicentric juvenile pilocytic astrocytoma. J Neurosurg. 1994;81: 24-30.

2. Pollack IF, Hurtt M, Pang D, Albright AL. Dissemination of low grade intracranial astrocytomas in children. Cancer. 1994;73: 2869-2878.

3. Civitello LA, Packer RJ, Rorke LB, Siegel K, Sutton LN, Schut L. Leptomeningeal dissemination of low-grade gliomas in childhood. Neurology. 1988;38:562-6.

4. Aichholzer M, Mazal PR, Haberler C, et al. Epidural metastasis of a glioblastoma after stereotactic biopsy: case report. Minim Invasive Neurosurg. 2001;44:175-7.
5. Perilongo G, Garrè ML, Giangaspero F. Low-grade gliomas and leptomeningeal dissemination: a poorly understood phenomenon. Childs Nerv Syst. 2003;19:197-203.

6. Gnekow AK, Falkenstein F, von Hornstein S, et al. Long-term follow-up of the multicenter, multidisciplinary treatment study HIT-LGG-1996 for low-grade glioma in children and adolescents of the German Speaking Society of Pediatric Oncology and Hematology. Neuro Oncol. 2012;14:1265-84.

7. McLaughlin JE. Juvenile astrocytomas with subarachnoid spread. J Pathol. 1976;118:101-7.

8. Ater JL, Zhou T, Holmes E, et al. Randomized study of two chemotherapy regimens for treatment of low-grade glioma in young children: a report from the Children's Oncology Group. J Clin Oncol. 2012;30:2641-7. 